PAIN

\title{
Silencing chronic pain with botulinum toxin
}

Chronic pain is extremely difficult to manage, with the use of currently available drugs limited by side effects, the development of tolerance and abuse liability. Writing in Science Translational Medicine, Hunt, Davletov and colleagues now demonstrate that a single injection of novel botulinum neurotoxin (BoNT) conjugates provides long-lasting pain relief in mouse models of inflammatory and neuropathic pain, without adverse effects.

BoNT serotype A (BoNT-A), produced by the anaerobic bacterium Clostridium botulinum, is made up of a light-chain zinc endopeptidase and a heavy chain that binds the toxin to neuronal receptors and promotes essential light-chain translocation across the endosomal membrane. Once internalized within the neuron, the light chain performs specific proteolytic cleavage of synaptosomal associated protein 25 (a protein involved in the regulation of neurotransmitter release), thereby silencing neurons. BoNT-A has been approved for the treatment of chronic migraines for several years and is being widely investigated for the treatment of other pain conditions.

The authors set out to exploit the neurosilencing properties of BoNT-A to specifically block key painprocessing neurons - neurokinin 1 receptor $\left(\mathrm{NK}_{1} \mathrm{R}\right)$-expressing neurons that relay pain-related information from the spinal cord to the brain and the $\mu$-opiate receptor ( $\mu \mathrm{OR})$ expressing neurons that modulate activity of $\mathrm{NK}_{1} \mathrm{R}$-expressing output neurons - in the spinal cords of mice.

To do this, Davletov's laboratory applied a protein-stapling method using SNARE (soluble $\mathrm{N}$-ethylmaleimide-sensitive factor attachment protein receptor) complex proteins to link the light-chain/ translocation domain of BoNT-A to the neuropeptide ligands substance $P$ (SP) and dermorphin (Derm). The resulting constructs, SP-botulinum (SP-BOT) and Derm-botulinum (Derm-BOT), aimed to silence the $\mathrm{NK}_{1} \mathrm{R}$-expressing and $\mu \mathrm{OR}$-expressing neurons, respectively.

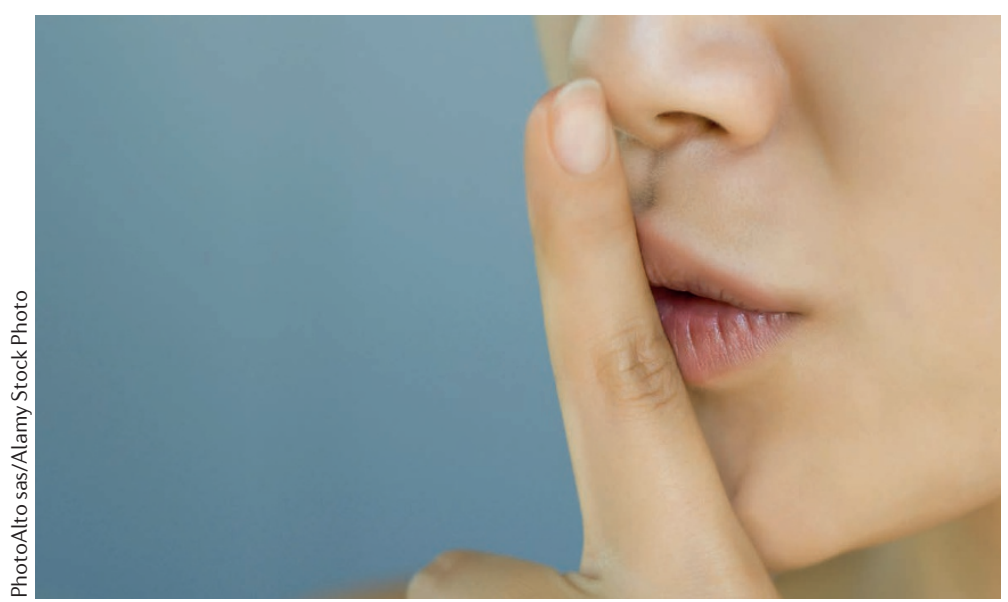

In studies led by Maiaru, Hunt's laboratory then tested the efficacy of the constructs in mouse pain models. In two mouse models of inflammatory pain induced by ankle or hind paw injection of complete Freund's adjuvant, a single intrathecal injection of the SP-BOT conjugate substantially reduced mechanical hypersensitivity for the duration of the experiment (21 days for the ankle model and 12 days for the hind paw model).

SP-BOT was also effective in controlling neuropathic pain. In the unilateral spared nerve injury (SNI) model of neuropathic pain, intrathecal injection of SP-BOT reduced mechanical hypersensitivity, with effects beginning around 3 days after SP-BOT injection and lasting for the 22 days of the experiment. This alleviation of pain was not seen in $N k 1 r^{-1-}$ mice.

A single intrathecal injection of Derm-BOT similarly reduced mechanical hypersensitivity in the mouse models of inflammatory and neuropathic pain that lasted until the end of the experiments (18 and 23 days, respectively). Notably, Derm-BOT reduced mechanical sensitivity in SNI mice to the same extent as that achieved with intrathecal morphine.

Importantly, both SP-BOT and Derm-BOT were shown to be specific, binding to and being internalized only by $\mathrm{NK}_{1} \mathrm{R}$-expressing and $\mu \mathrm{OR}$-expressing neurons, respectively, without signs of toxicity.

In summary, this strategy may represent an effective approach for the long-term amelioration of chronic pain states, which may avoid the adverse events associated with existing therapies.

Sarah Crunkhorn

ORIGINAL ARTICLE Maiaru, M. et al. Selective neuronal silencing using synthetic botulinum molecules alleviates chronic pain in mice. Sci. Transl Med. 10, eaar7384 (2018)

FURTHER READING Yekkirala, A. et al. Breaking

barriers to novel analgesic drug development.

Nat. Rev. Drug Discov. 16, 545-564 (2017) 